\title{
Magnetic field evolution in a quiet region and a coronal hole
}

\author{
Jun Zhang \\ National Astronomical Observatories, Chinese Academy of Sciences, Beijing 100012, China \\ email: zjun@ourstar.bao.ac.cn
}

\begin{abstract}
Using Big Bear Solar Observatory (BBSO) magnetograms and $\mathrm{H} \alpha$ images in a quiet region and a coronal hole, observed on September 14 and 16, 2004, respectively, we have explored the magnetic flux emergence, disappearance and distribution in the two regions. The following results are obtained: (1) The evolution of magnetic flux in the quiet region is much faster than that in the coronal hole, as the flux appeared in the form of ephemeral regions in the quiet region is four times as large as that in the coronal hole, and the flux disappeared in the form of flux cancellation, three times as fast as in the coronal hole. (2) More magnetic elements with opposite polarities in the quiet region are connected by arch filaments. (3) The flux distribution of network and intranetwork (IN) elements is similar in both polarities in the quiet region. For network fields in the coronal hole, the number of negative elements is much more than that of positive elements. However for the IN fields, the number of positive elements is much more than that of negative elements. (4) In the coronal hole, the fraction of negative flux change obviously with different threshold flux density. $73 \%$ of the magnetic fields with flux density larger than 2 Gauss is negative polarity, and $95 \%$ of the magnetic fields is negative, if we only measure the fields with their flux density larger than 20 Gauss. These results display that in a coronal hole, stronger fields is occupied by one predominant polarity; however the majority of weaker fields, occupied by the other polarity.
\end{abstract}

Keywords. Sun: chromosphere — Sun: magnetic fields — Sun: UV radiation

\section{Introduction}

Coronal holes are cool, low density regions observed both at low latitudes and at the two polar regions of the Sun (Chiuderi Drago et al. 1999). They were first observed on X-ray plates by Underwood \& Muney(1967), on EUV line spectroheliograms by Reeves \& Parkinson(1970) and in white light by Altshuler \& Perry(1972). Their predominantly unipolar magnetic fields are open to the interplanetary region (Bohlin 1977), giving rise to high-speed solar wind that can lead to geomagnetic storms (Krieger \& Timothy 1973). There are 3 broad categories of coronal holes: polar, non-polar and transient. Polar coronal holes have long lifetimes (about 8 years). Non-polar coronal holes are usually associated with remnants of active regions and may persist for many solar rotations. Transient coronal holes are associated with eruptive events like filament eruptions and coronal mass ejections (CMEs) and have lifetimes of several days (Harvey \& Recely 2002). Low latitude coronal holes may show quasi-rigid rotation and it has been suggested that magnetic reconnection must occur continuously at the boundary in order to maintain the coronal hole integrity (Kahler \& Hudson 2002).

In order to solve some of the key problems of solar and stellar physics, such as the coronal heating and solar wind acceleration, we need to study and understand the smallscale magnetic activity in coronal holes and quiet regions. In this paper, we focus on two topics. The first is dipole flux emergence (Wang, Wang, \& Shi 1992) and flux disappearance in a coronal hole and a quiet region, and the second, distribution of magnetic flux 
with both polarities in the two observing regions, using BBSO deep magnetograms with the noise level of 2 Gauss. This may help us to understand the nature of the different magnetic properties between quiet regions and coronal holes.

\section{Observations and analysis}

From 2004 September 13 to 18, campaign observations were arranged between BBSO and the Transition Region and Coronal Explorer (TRACE) teams. We used the data obtained on September 14 and 16 for this study as the two days had rather good seeing. The observational target on 14 was a quiet region centered at N9W13, and on 16, a coronal hole at N31E14. $\mathrm{H} \alpha \pm 0.60 \AA$, Ca II K line, and magnetograms were observed simultaneously, using 25 and $65 \mathrm{~cm}$ telescopes at BBSO. Images at $\mathrm{H} \alpha \pm 0.60 \AA$ were taken with an Orbiting Solar Laboratory (OSL) CCD camera mounted on the $65 \mathrm{~cm}$ telescope with a $30 \mathrm{~s}$ cadence. The FOV is $210^{\prime \prime} \times 210^{\prime \prime}$, and the spatial resolution is $0^{\prime \prime} .4 \mathrm{pixel}^{-1}$. For magnetic field observations, we used the digital vector magnetograph (DVMG) system on the same $25 \mathrm{~cm}$ telescope. The DVMG system uses liquid crystal, a Zeiss filter, and a 12 bit digital camera so that one can accurately measure small intranetwork magnetic elements on the order of $2 \mathrm{G}$. The temporal resolution is $90 \mathrm{~s}$, and the FOV is $300^{\prime \prime} \times 300^{\prime \prime}\left(0^{\prime \prime} .6 \mathrm{pixel}^{-1}\right)$.

The high-resolution UV observation was obtained from TRACE satellite. TRACE offers an unprecedented opportunity to learn the fine structure and dynamics of the corona and transition region. The $1600 \AA$ images used in this study have spatial resolution of $1^{\prime \prime} .0$, temporal resolution of $40 \mathrm{~s}$, and field-of-view of $250^{\prime \prime} \times 250^{\prime \prime}$.

\section{Results}

\subsection{Magnetic flux emergence and disappearance}

In the co-spatial field-of-view (about $200 \times 200$ square arcsec) among BBSO magnetograms, H $\alpha$ filtergrams, and TRACE $1600 \AA$ images, We identified 30 pairs of ephemeral regions (ERs) in the quiet region on September 14, 2004 during the 7-hour observations. The mean flux of an ER is $8.1 \times 10^{18} \mathrm{Mx}$. 7 of the $30 \mathrm{ERs}$ are connected by arch filaments, seeing from $\mathrm{H} \alpha$ images. We find just as the appearance of an ER, UV bright points always appear at the feet of the two magnetic elements, seen from TRACE 1600 $\AA$ images. For the coronal hole, although the observational time and the field-of-view are the same as that of the quiet region, we can only track 17 pairs of ERs, and one of the $17 \mathrm{ERs}$ is connected by an arch filament. The mean flux of an ER is $3.4 \times 10^{18} \mathrm{Mx}$, much smaller than that in the quiet region. This implies that the evolution of magnetic flux in the coronal hole is much slower than that in the quiet region. We find that only if the flux is larger than $5.0 \times 10^{18} \mathrm{Mx}$ and flux density higher than 20 Gauss then the ERs are observed to be connected by arch filament systems. Considering ERs as closed magnetic loops, we can estimate the flux density of these loops ranges from 6 to 40 Gauss in the quiet region, and from 6 to 22 Gauss in the coronal hole.

Another parameter which can also represent the magnetic evolution rate is magnetic flux disappearance. The disappearance is mainly due to the cancellation of opposite polarity elements. If an magnetic element meets with another of opposite polarity, they can cancel each other. Under this condition, the total flux decreases. In the quiet region, about $1.7 \times 10^{20} \mathrm{Mx}\left(2.1 \times 10^{20} \mathrm{Mx}\right)$ positive flux (negative flux) disappear by the cancellation in the 7 -hour observations. In the coronal hole, the amount of the disappeared flux is about $3.5 \times 10^{19} \mathrm{Mx}\left(9.6 \times 10^{19} \mathrm{Mx}\right)$ for positive flux (negative flux), one third as large as that in the quiet region. 

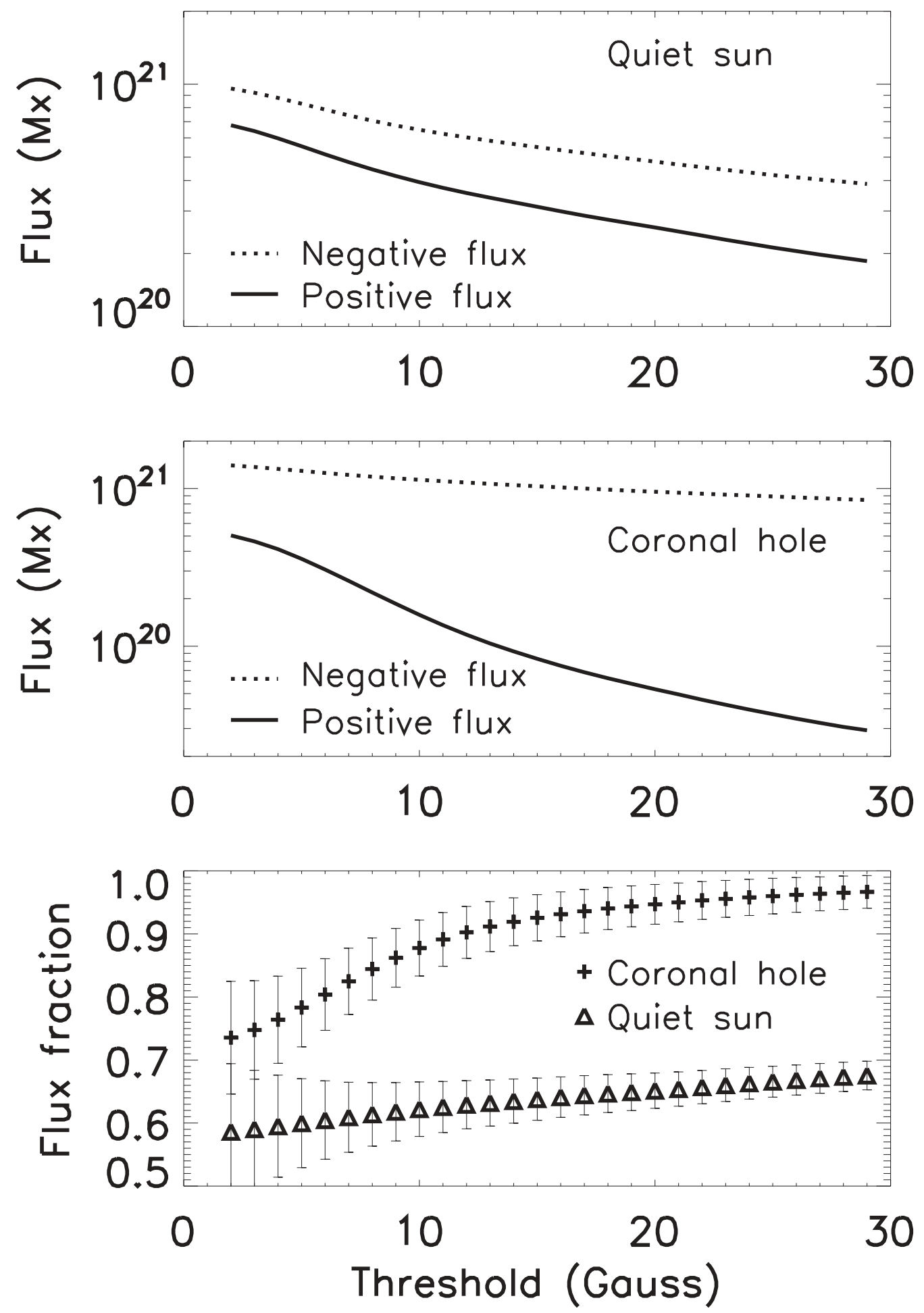

Figure 1. Variation of magnetic flux vs threshold flux density in the field-of-view of the quiet region (upper) and the coronal hole (middle). The lower panel displays the flux fraction of the negative flux relative to the total flux. 


\subsection{Magnetic flux distribution}

It is always suggested that coronal holes lie within a unipolar magnetic region. However the solar magnetic field is never strictly unipolar. We have no knowledge about the IN flux distribution in coronal holes, although Wang et al. (1995) presented flux distribution of IN and network magnetic elements in a quiet region. Here we study the flux distribution in both polarities in the coronal hole, and compare with the quiet region. Three criteria are used to separate the IN elements from the network elements (Wang et al. 1995). For each region, about 1000 magnetic elements are identified and measured. In the quiet region, the flux distribution of IN and network elements is similar in both polarities. For network fields in the coronal hole, there is no positive elements in the range where magnetic flux larger than $4.0 \times 10^{18} \mathrm{Mx}$. However for the IN fields, the number of positive elements is much larger than that of negative elements. In other words, in this coronal hole the stronger field is occupied by one predominant polarity; and the majority of weaker fields, occupied by the opposite polarity.

Now we measure the magnetic flux in both polarities instead of the individual elements in the two regions. Figure 1 shows the variation of magnetic flux versus threshold flux density in the field-of-view of the quiet sun and the coronal hole. As the threshold flux density increases from 2 Gauss (near noise level) to 30 Gauss, the flux in both polarities decreases homologically in the quiet region (see the upper panel of Figure 1). In the coronal hole, the positive flux decreases more quickly than the negative flux with increasing threshold flux density, as shown in the middle panel of Figure 1. The bottom panel presents the fraction of the negative flux to the total (negative plus positive) flux. In the coronal hole, $73 \%$ of the magnetic fields with flux density larger than 2 Gauss is negative, and $95 \%$ of the magnetic fields is negative, if we only measure the fields with their flux density larger than 20 Gauss, the noise level of a typical magnetogram. Comparing to the coronal hole, we conclude that the fraction in the quiet region changes more gently. This result further confirms the conclusion that in the coronal hole, stronger field is occupied by one predominant polarity, and weaker fields, occupied by the opposite polarity with a large margin.

\section{Acknowledgements}

JZ is indebted to the TRACE and BBSO teams for providing the wonderful data. The work is supported by the National Natural Science Foundations of China (G10573025 and G10233050), the State Ministry of Science and Technology of China under Grant of G200078404.

\section{References}

Altshuler, M. D., \& Perry, R. M. 1972, Solar Phys. 23, 410

Bohlin, J. D. 1977, Solar Phys. 51, 377

Chiuderi Drago, F., Landi, E., Fludra, A., \& Kerdraon, A. 1999, A $\mathscr{E} A$ 348, 261

Harvey, K. L., \& Recely, F. 2002, Solar Phys. 211, 31

Kahler, S. W., \& Hudson, H. S. 2002, ApJ 574, 467

Krieger, A. S., \& Timothy, A. F. 1973, Solar Phys. 29, 505

Reeves, E. M., \& Parkinson, W. H. 1970, ApJS 21, 1

Underwood, J. H., \& Muney, W. S. 1967, Solar Phys. 1, 129

Wang, J., Wang, H., \& Shi, Z. 1992, ASP Conference Series 27, 108

Wang, J., Wang, H., Tang, F., et al. 1995, Solar Phys. 160, 277 